Designing and Implementing Emergency Department Pain Management Curriculum: A Delphi Approach

\author{
Karolina Paziana, MD, Edmund Timpano, MD, and Andrew Stolbach, MD, MPH
}

\begin{abstract}
Objectives: Our primary objective was to use the Delphi method (DM) to choose learning objectives for an Internetbased pain management teaching module for emergency care providers. The DM is a structured communication technique that uses systematic, interactive data gathering to reach a consensus among a panel of experts.

Methods: We extracted preliminary educational objectives from nationwide pain fellowship training programs. After redundant objectives and those relating to procedures outside the scope of emergency medicine (EM) were removed, 23 preliminary objectives remained. We enlisted experts in EM, medical toxicology, anesthesia, pain, psychiatry, and medical education to evaluate the objectives in repeated rounds. Based on the time we had designated for teaching ( 3 hours) and our estimate of 15 to 20 minutes for each learning objective, we aimed for nine to 12 final learning objectives. The expert panel rated the 23 preliminary objectives with a 5 -point Likert scale. Experts were blinded to each other's answers. When $80 \%$ of experts agreed with proposed objectives, objectives were considered "accepted" and retained. When $80 \%$ of experts disagreed with proposed objectives, the objectives were considered "rejected" and discarded. The remaining objectives were considered in subsequent rounds. In the first round, one objective was rejected and four were accepted. In the second round, four objectives were rejected and five were accepted. Having reached nine objectives the panel unanimously agreed that the nine objectives were sufficient to be used as a foundation for teaching modules.
\end{abstract}

Results: Using the DM, our expert panel identified nine educational objectives that were used as the foundation for an educational module. Of the nine objectives, three were categorized as "medical knowledge," two as "pharmacologic knowledge," two as "patient communication," and two as "systems-based practice." The learning objectives are as follows: recognize common barriers to treating pain; understand alternative interventional therapies used to treat different kinds of pain their implications, benefits, and risks; identify common component symptoms that may exacerbate chronic pain (i.e., depression, anxiety, delirium, cachexia, dyspnea); study the major drug groups used to treat different kinds of pain; recognize the risks of polypharmacy; set realistic expectations with patients early in their presentation to the emergency department (ED); educate patients as the risks of different classes of pain medications, including risk of addiction; understand the legal and regulatory issues that govern pain management in the ED; and understand in-hospital resources available for patient follow up and referral to pain clinics.

Conclusions: We successfully used the DM to develop educational objectives for an e-learning module. Further work should evaluate educational outcomes of the teaching module.

$\mathrm{O}$ ver 100 million Americans suffer from pain, a quarter of whom report either moderate or severe disability. ${ }^{1}$ Of the 136 million annual U.S. emergency department (ED) visits, 75\% have a chief complaint related to pain. ${ }^{2,3}$ Pain limits quality of life, productivity, and social relationships. Ineffective pain

From the Johns Hopkins University (KP, ED, AS), Baltimore, MD. Dr. Paziana is currently with the Mercy Medical Center, Redding, CA. Dr. Timpano is currently with the St. Agnes Hospital, Baltimore, MD.

Received September 30, 2017; revision received January 10, 2018; accepted January 28, 2018.

The authors have no relevant financial information or potential conflicts to disclose.

Supervising Editor: Rebecca Blanchard, PhD.

Address for correspondence and reprints: Andrew Stolbach, MD, MPH; e-mail: astolba2@jhmi.edu.

AEM EDUCATION AND TRAINING 2018;2:121-129 
management results in financial hardship both on the affected individual and on society as a whole, costing an estimated $\$ 560$ to $\$ 630$ billion a year in missed work and medical expenses. ${ }^{4}$ Inappropriate prescribing and nonmedical use of prescription opioids has contributed to the current North American opioid epidemic. $^{5}$

Emergency medicine (EM) trainees may arrive from medical school with knowledge gaps in management of pain. A survey evaluating physician, nurse, pharmacist, and student knowledge regarding pain management, only $56 \%$ of questions were answered correctly. ${ }^{6}$ ED studies demonstrate inconsistency in practice standards and identify inadequate tools and provider education as a key factor in pain mismanagement. ${ }^{7}$

Currently, there is no universal pain management curriculum among EM training programs. The Model of the Clinical Practice of Emergency Medicine (the Model) and The Emergency Medicine Milestones Project provide some framework, however. ${ }^{8,9}$

Residencies preparing trainees for eventual American Board of Emergency Medicine (ABEM) certification in EM follow the Model of the Clinical Practice of Emergency Medicine. ${ }^{8}$ The Model, reviewed by the EM Model Review Task Force every 3 years, defines the content of the ABEM board examination, which is required for EM board certification. In this way, the Model delineates the scope of EM practice. In the Model, several types of pain are identified as "signs, symptoms, and presentations" to be tested (see Table 1). However, the Model does not specify how particular presentations should be taught or frame these presentations as learning objectives. The Model does not mention communication or systems-based practice aspects of pain management. In a separate "toxicologic disorders" section, the Model also mentions opioids, nonsteroidal anti-inflammatories, and

Table 1

Types of Pain in EM Model of the Clinical Practice of EM ${ }^{8}$

\begin{tabular}{lccc}
\hline Type & Critical & Current & Lower Acuity \\
\hline Pain (unspecified) & $\mathrm{X}$ & $\mathrm{X}$ & $\mathrm{X}$ \\
\hline Headache & $\mathrm{X}$ & $\mathrm{X}$ & $\mathrm{X}$ \\
\hline Eye pain & & $\mathrm{X}$ & $\mathrm{X}$ \\
\hline Chest pain & $\mathrm{X}$ & $\mathrm{X}$ & $\mathrm{X}$ \\
\hline Abdominal pain & $\mathrm{X}$ & $\mathrm{X}$ & $\mathrm{X}$ \\
\hline Pelvic pain & $\mathrm{X}$ & $\mathrm{X}$ & $\mathrm{X}$ \\
\hline Back pain & $\mathrm{X}$ & $\mathrm{X}$ & $\mathrm{X}$ \\
\hline Chronic pain & & & $\mathrm{X}$ \\
\hline Extremity pain & $\mathrm{X}$ & $\mathrm{X}$ & $\mathrm{X}$ \\
\hline
\end{tabular}

acetaminophen. However, these medications are presented in the context of toxicity, not therapeutic use. Although the Model largely defines the content of EM training curricula, it does not provide educational objectives nor does it provide guidance on how to teach the content.

The Accreditation Council of Graduate Medical Education (ACGME) presents a skill developmental framework shared by accredited U.S. EM programs known as the EM Milestones. 9 The document provides a framework to evaluate residents using competency-based developmental outcomes. The framework consists of 23 distinct subcompetencies identified by ACGME and ABEM. Each subcompetency is divided into five proficiency levels (1-5), which are, in turn, composed of competency-based "milestones." Residency directors are expected to track individual resident's advances in proficiency level, measured by mastering milestones, in the subcompetencies.

One subcompetency, "anesthesia and pain management" addresses pain management, anesthesia, and procedural sedation. Within this subcompetency there are 11 milestones. Four of these milestones address local and regional anesthesia, two address doses of analgesic medications, four address procedural sedation, and one describes development of pain management protocols and care plans (see Table 2). Although the purpose of the milestones is to track development, there is no standard measure to evaluate progress in these subcompetencies; training programs are at liberty to choose their own measures.

Currently, many programs use end-of-shift evaluations or self-evaluations to measure proficiency in these milestones. Unfortunately, these two types of evaluation may not accurately reflect the residents' proficiency level. ${ }^{10,11}$ Both types of measures tend to overestimate proficiency level. Therefore, the EM Milestones describe pain management competencies that residents should achieve without providing corresponding individual educational objectives to achieve these objectives or mechanisms of measuring progress in these competencies.

There are no Internet-based educational resources on pain specifically targeted to emergency providers. Although there are online resources targeted toward medical and nursing students, there remains a lack of resources oriented toward acute and EM trainees and providers. ${ }^{12,13}$ In light of this gap, we aimed to write an Internet-based module on the topic of pain assessment and management strategies for the emergency 
Table 2

ACGME EM Milestones Relevant to Pain Management ${ }^{9}$

\begin{tabular}{|c|c|c|}
\hline Category & Milestone & $\begin{array}{l}\text { Proficiency } \\
\text { Level }\end{array}$ \\
\hline \multirow{3}{*}{$\begin{array}{l}\text { Local and } \\
\text { regional } \\
\text { anesthesia }\end{array}$} & Discusses with the patient indications, contraindications, and possible complications of local anesthesia. & 1 \\
\hline & $\begin{array}{l}\text { Performs local anesthesia using appropriate doses of local anesthetic and appropriate } \\
\text { technique to provide skin to subdermal anesthesia for procedures. }\end{array}$ & 1 \\
\hline & $\begin{array}{l}\text { Knows the anatomic landmarks, indications, contraindications, potential complications, } \\
\text { and appropriate doses of local anesthetics used for regional anesthesia. }\end{array}$ & 2 \\
\hline $\begin{array}{l}\text { Analgesic/ } \\
\text { sedative } \\
\text { medications }\end{array}$ & $\begin{array}{l}\text { Performs patient assessment and discusses with the patient the most appropriate analgesic/sedative } \\
\text { medication and administers in the most appropriate dose and route. }\end{array}$ & 3 \\
\hline $\begin{array}{l}\text { Procedural } \\
\text { sedation }\end{array}$ & $\begin{array}{l}\text { Knows the indications, contraindications, potential complications, and appropriate doses of } \\
\text { medications used for procedural sedation. }\end{array}$ & 3 \\
\hline $\begin{array}{l}\text { Protocols and } \\
\text { care plans }\end{array}$ & Develops pain management protocols/care plans. & 5 \\
\hline
\end{tabular}

ACGME $=$ Accreditation Council of Graduate Medical Education .

care provider and to describe the process used to develop this content. The focus of our module would be types of painful presentation common to emergency care providers, including acute pain and acute exacerbations of chronic pain. We did not address management of longitudinal management of chronic pain or procedures in management of pain. We focused on clinical, as opposed to biochemical or theoretical aspects of pain. We chose this clinical approach, rather than a theoretical approach, because of literature supporting the use of relevant objectives and credible clinical scenarios as best practices in self-directed learning. ${ }^{14,15}$ Resident physicians have many competing demands for time and should accept training that helps them solve clinical challenges at work.

\section{Delphi Method}

The Delphi method (DM) is a structured communication technique that uses systematic, interactive data gathering to reach a consensus among a panel of experts. The DM was originally developed to incorporate expert opinion into policy decision making by the RAND Corporation after World War II. ${ }^{16}$ Since that time, the same underlying principles of the DM have evolved from exclusive use in military forecasting to use in business management and, most recently, medical consensus.

There is no single Delphi methodology, but Delphi processes share several principles. ${ }^{17}$ The process begins with enlistment of experts. No specific number of experts is prescribed, as long as they can address the question. Next, experts are surveyed in two or more rounds. In between rounds, experts are given the opportunity to incorporate results from prior rounds. ${ }^{18,19}$ Experts are often anonymous to each other to minimize bias, but a modified Delphi technique has been described that incorporates in-person meetings at certain stages of the process to encourage consensus.

A 2012 systematic review of all 80 reports of the use of the DM development for health care quality indicators from 1978 to 2009 presented recommendation for strengthening the DM. ${ }^{18}$ These recommendations were used to execute the DM used to gather expert consensus for our education model on acute care pain management.

1. Experts: Gather a heterogeneous panel and ask if they are willing to participate. The composition and characteristics of the panel should be reported.

2. First round: define the study objectives and what is expected of the panelists. The method of the question selection and definition of consensus should be reported.

3. Questionnaires: should be sent by internet or mail.

4. Subsequent rounds: Construct based on the results of the prior rounds and exclude measures for which there was no consensus. Report group median and qualitative feedback about prior rounds 
and report which questions were added and which were removed.

5. Final round: End the Delphi process when a consensus is reached. If a consensus cannot be reached a physical meeting should be arranged. The number of rounds should be reported.

\section{METHODS}

Our objective was to enlist experts and use the DM to reach consensus on pain management learning objectives for EM trainees. We used educational objectives derived from the DM to write self-directed e-learning modules. This project is considered to be "exempt research" by the Johns Hopkins Hospital Institutional Review Board.

\section{DM}

We assembled a list of individuals with expertise in one or more areas relevant to medical education or treating pain in the acute setting. We determined that the most relevant disciplines included anesthesia, EM, medical education, medical toxicology, pain medicine, and psychiatry. Prospective experts were chosen from authors of scholarship in the topic and the professional network of investigators. An e-mail was sent to five candidates detailing the Delphi process, a brief description of the project and its objectives, the expected response timeline for each round, and expected time commitment. Prospective experts were given the opportunity to decline participation and to name other potential experts that could substitute.

Next, we used our expert panel to reach consensus on educational objectives. One of our investigators (KP) served as "moderator" to coordinate the process. Neither the moderator nor any of the other investigators served as experts.

We extracted the proposed educational from educational goals from a convenience sample of 12 nationwide pain fellowship training programs. When educational goals were not formatted as educational objectives we rewrote them. When curricula were not available online, we contacted program administrators and directors. This process yielded a total of over 100 potential core areas of focus. We removed redundant objectives and objectives related to procedures outside the scope of EM. The remaining 23 preliminary objectives were organized under the following headings: medical knowledge, pharmacologic knowledge, patient communication, and systems-based practice.

The moderator distributed the list of proposed objectives to the expert panel anonymously via Google Forms (Alphabet Inc.). Expert panelists remained blinded to each other's survey results during the feedback process. Following each round, experts could only see aggregate survey results. We aimed to identify nine to 12 learning objectives for our teaching module.

The panelists were asked to rate their preference for the inclusion of each proposed objective in the module via a 5-point Likert scale from "strongly disagree" to "strongly agree." Consensus was defined as expert consensus on agreement (agree and strongly agree) or disagreement (disagree and strongly disagree) of $80 \%$. Any proposed objective with expert agreement of $80 \%$ or greater was "accepted" and added to the final list of objectives. If a proposed objective received a score of $80 \%$ for "disagree" (disagree or strongly disagree) it was "rejected" and removed from the list. The remaining proposed objectives that did not reach consensus $(80 \%$ agreement or disagreement) would be retained for the next round. The panelists were also given the opportunity to add new objectives to each round. We planned for this iterative process to be repeated round after round until an $80 \%$ consensus of agreement would be reached on nine to 12 objectives. Following consensus on objectives, experts were asked if the final objectives were sufficient and appropriate to teach the topic.

\section{RESULTS}

\section{Delphi Results}

In the first part of the study we engaged experts to reach consensus on educational objectives. We invited six experts representing six different disciplines with expertise in acute pain management or medical education. Of the six prospective candidates contacted, five accepted. Each expert that accepted had expertise in more than one discipline. The characteristics of the experts are listed in Table 3.

Four proposed objectives reached $80 \%$ or greater agreement in Round 1 and were therefore adopted. One proposed objective reached $80 \%$ or greater disagreement in Round 1 and was discarded. The aggregate results from Round 1 were shared with the panel and the remaining proposed objectives were considered in Round 2. The results of Round 1 are demonstrated in Table 4. 


\begin{tabular}{|c|c|c|c|}
\hline \multicolumn{4}{|c|}{$\begin{array}{l}\text { Table } 3 \\
\text { Characteristics of Panel of Experts }\end{array}$} \\
\hline Expert & Area of Expertise & Years As Attending & Sex \\
\hline \multirow[t]{2}{*}{ A } & Anesthesiology & $>20$ & Male \\
\hline & Pain medicine & & \\
\hline \multirow[t]{2}{*}{ B } & Pain medicine & $>20$ & Male \\
\hline & Psychiatry & & \\
\hline \multirow[t]{2}{*}{ C } & EM & $5-10$ & Female \\
\hline & Medical toxicology & & \\
\hline \multirow[t]{2}{*}{$\mathrm{D}$} & EM & $>20$ & Male \\
\hline & Medical toxicology & & \\
\hline \multirow[t]{2}{*}{$E$} & EM & $11-20$ & Female \\
\hline & Medical education & & \\
\hline
\end{tabular}

During Round 2, the objectives that did not meet consensus were sent back to the experts. The same Likert scale was used to evaluate the objectives. Consensus was again defined as $80 \%$ agreement or disagreement. In Round 2, five proposed objectives reached $80 \%$ agreement and were adopted, while four proposed objectives reached $80 \%$ disagreement and were discarded. The results of Round 2 are demonstrated in Table 4. A consensus of nine objectives was achieved during Round 2. We returned the nine adopted objectives to panel members, who unanimously agreed that they were sufficient to teach the topic. The nine module objectives that received $80 \%$ agreement are listed in Table 5. The objectives were then used as the backbone for an e-learning module on emergency pain management.

\section{DISCUSSION}

We used the DM to engage a panel of experts to reach a consensus on nine educational objectives relevant to ED pain management. We aimed to identify no more than 12 objectives, based on an administrative limit of 3 hours of resident instructional time and allotting at least 15 minutes per objective. Our trainees are required to perform a certain amount of self-study, and we designated 3 hours for that requirement. Increased educational time results in improved educational outcomes. ${ }^{14}$ However, learners can only maintain focus for a limited amount of time and have similar educational outcomes performing multiple brief sessions, compared to fewer long sessions, when instructional time is held constant. ${ }^{15}$ We wanted to maximize total instructional time while dividing the module into digestible pieces. The optimal length of an e-learning instructional session depends on the interactivity of the material, learner preference, and the relevance of the material to the learner. ${ }^{14}$ From our experience, our learners prefer to spend no more than 15 to 20 minutes for a single e-learning session. For structural clarity, we aimed for each e-learning session to correspond to a single learning objective. By setting a total instructional time of 3 hours, using nine to 12 objectives would provide 15 to 20 minutes per objective.

Nine objectives were accepted based on $80 \%$ or greater expert consensus (Table 5). Experts accepted at least two objectives from each heading: medical knowledge, pharmacological knowledge, patient communication, and systems-based practice. The objectives identified by our experts contrasted with the types of pain described by the EM Model (Table 1). Whereas our learning objectives describe issues related to therapies, patient factors, communication, and systems issues, the EM Model merely divides pain presentations into anatomical regions-e.g., "headache," "eye pain," etc.

Our objectives differed in focus from the 11 painrelated developmental proficiencies identified by the Emergency Medicine Milestones Project (Table 2). Four of our nine objectives related to patient communication or systems-based practice. In contrast, the EM Milestones has only one proficiency that aligns with the communication or system-based practice: "develops pain management protocols/care plans." Another major difference was in procedural sedation. The Milestones identified four proficiencies related to performing procedural sedation, whereas we had none. This is understandable, since we focused our modules on treatment of acute exacerbations of chronic painful conditions. In contrast, procedural sedation is typically used for performing painful procedures.

Five objectives were rejected based on $80 \%$ or greater expert consensus (Table 4). In Round 1, "learn the appropriate dosing of each analgesic" was rejected. In Round 2, experts rejected "teach the pathophysiology of different types of pain," "identify Beers list medications," "understand the benefits and risks of local anesthetic procedures (neurolytic blocks, implanted drug delivery systems)," and "discuss the role of various heath professionals in the management of pain." We did not survey experts for justification of their choices, so we do not know why objectives were rejected. Compared with the adopted objectives, the rejected objectives tended to focus more on facts requiring rote memory and information with more 


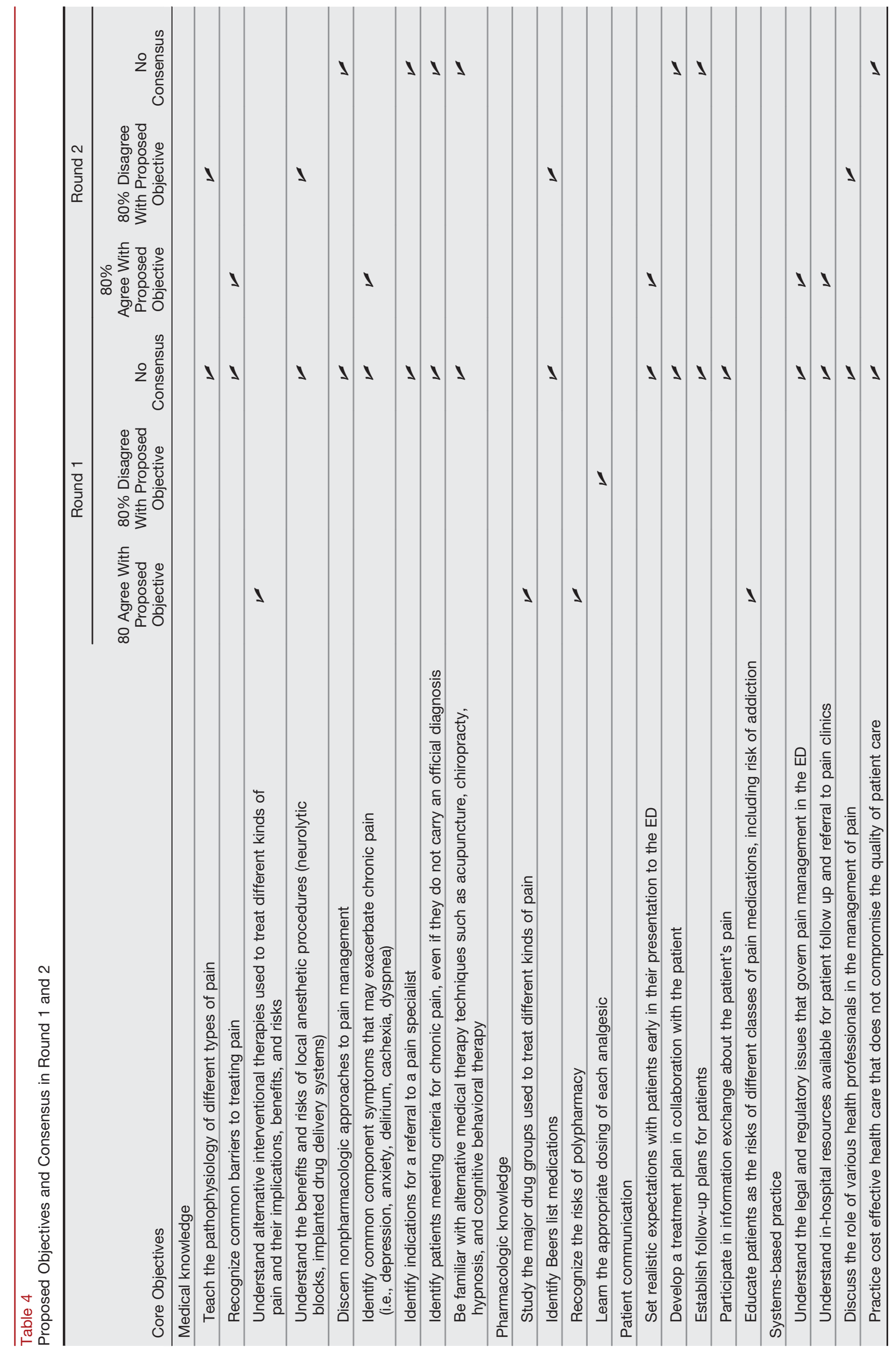


Table 5

Final List of Educational Objectives Derived by Delphi Consensus

Equal to or greater than $80 \%$ consensus on objectives

1. Discussion of common barriers to effective pain

management

2. Education of alternative therapies to pharmaceutical analgesia

3. Education on comorbidities that may exacerbate chronic pain

4. Education on how to identify and educate patients on prescription drug use, misuse, and abuse

5. Descriptions of the different classes of analgesics used to treat different kinds of pain

6. Education on the common adverse effects and interactions of commonly used analgesics

7. Instruction to set realistic expectations with patients early in their presentation

8. Discussion of the legal and regulatory issues that govern pain management in the acute care setting

9. List hospital resources and referral process for appropriate pain management

scientific than clinical relevance. As an example, the rejected objective "learn the appropriate dosing of each analgesic" would require memorizing dozens of pain medications. Learning the appropriate dose of each one may be unrealistic and less safe than simply looking these up or relying on computer decision support. In their choice of objectives, our experts seem to have valued clinically relevant concepts over memorization of facts

Nine more objectives were not used because experts could not reach consensus (Table 4). Again, we did not survey experts as to why they did not choose objectives. Two objectives without consensus related to alternative therapies and nonpharmacologic therapies. This lack of consensus may illustrate a disagreement among experts whether there is a role in the ED for treatments such as hypnosis or cognitive behavioral therapy. Three of the objectives with no consensus discussed aspects of forming treatment plans with patients and discussing their pain with them. Our experts apparently could not reach consensus on the role of emergency physician in developing longer-term treatment plans.

The DM enabled us to incorporate input from experts in several medical fields with different perspectives on pain. As is common with the DM, experts were anonymous to each other to minimize bias. By maintaining anonymity, experts could not defer to friends or senior colleagues as might occur in a live meeting. After each round, experts saw aggregate results from the group, but these results did not have personal identifiers. By including experts in several medical fields we were able to evaluate objectives that may not be commonly considered by any one specialty. We enlisted five experts. The DM does not specify a maximum or minimum number of experts, as long as the group is both heterogeneous and knowledgeable. Our five-person expert panel had two women, three men, and represented six specialties.

Using a moderator to manage the process ensured that experts saw aggregate, but not individual responses. We feel that this improved validity of the objectives by preventing experts from deferring to specific individuals on the panel. Experts were able to consider each other's ideas without the bias toward more senior or outspoken individuals that might occur in a face-to-face meeting. The use of iterative rounds allowed us to efficiently reach consensus through a structured group communication process. In only two rounds, we were able to reach consensus on nine objectives that all of our experts agreed was sufficient to teach the topic.

\section{LIMITATIONS}

Our preliminary objectives were drawn from a convenience sample of goals and objectives obtained from 12 fellowship programs. We did not obtain a comprehensive list of educational goals from all potential sources and we did not attempt to write new goals. Nevertheless, we feel that the preliminary objectives we obtained were sufficient because many of these were redundant. Of the more than 100 initial objectives, there were only 23 remaining after we eliminated duplicates and procedure-driven objective.

We limited the number of objectives at nine. This somewhat arbitrary figure was based on the amount of learner time that we had designated for module completion and an estimate of the appropriate amount of time to cover each objective. We aimed to create no more than 3 hours of overall resident self-study instructional time. In our experience, learners prefer to spend 15 to 20 minutes on a single e-learning session and course sessions should naturally correspond to learning objectives. Based on these parameters, we determined that nine to 12 objectives would be an appropriate number. At the conclusion of the Delphi exercise, our experts were presented the final list of objectives all agreed that they were sufficient to teach this topic. 
We only enlisted five experts to evaluate objectives. There is no agreement on the correct number of experts in a Delphi panel. For health care applications, panels from five to more than 1000 have been used. $^{20}$ In well-defined knowledge areas, small Delphi panels with good understanding of the field of interest have proven to be as reliable as larger panels. ${ }^{20}$ Although our panel was small, we feel that the knowledge area was well defined and our experts had excellent understanding of the subject. Therefore, we feel that the objectives agreed upon by our five experts were reliable.

The heterogeneity of our objectives may have been limited by expert disciplines represented in our panel. Our panel experts represented six disciplines: anesthesiology, EM, medical education, medical toxicology, pain medicine, and psychiatry. It is possible that we limited our results by failing to include experts from other disciplines such as physiatry or physical therapy. While there are many experts that address painful conditions, we felt that we chose experts in disciplines most closely related to teaching pain management in EM. Emergency physicians have first-hand knowledge of our patient population. Medical toxicologists and anesthesiologists have expertise in pharmacology. The psychiatrist on our panel specializes in mental health aspects of chronic pain. Our medical education expert was able to evaluate potential objectives from the standpoint of their educational appropriateness.

\section{CONCLUSION}

To our knowledge, this is the first application of the Delphi method to develop educational objectives for an e-learning module. We have described a structured process for engaging experts from diverse medical specialties to identify appropriate objectives for learners in emergency medicine. Engagement of other experts improved the quality of the objectives and allowed us to incorporate concepts that we would not have otherwise considered. The Delphi method may be a useful technique for employing experts from diverse specialties to develop educational programs for emergency residents.

Our experts chose objectives that focused nearly equally on medical knowledge, pharmacologic knowledge, systems-based factors, and patient communication. The panel eschewed objectives, such as specific medication dosing, that could easily be looked up or checked with decision support software. Our panel could not reach consensus on alternative therapies such as hypnosis and cognitive behavioral therapies.

There is currently no nationwide emergency medicine pain curriculum. The objectives we identified expand on the framework provided by the emergency medicine Model of the Clinical Practice of Emergency Medicine. The emergency medicine Model was not intended to provide learning objectives, but to delineate the scope of our expertise. As opposed to the EM Model, which only identifies anatomical regions of pain, our objectives discuss medical knowledge, pharmacologic knowledge, systems-based practice, and patient communication. Our objectives provide guidelines on how to manage pain-related presentations delineated by the Model.

Our learning objectives are complementary to the proficiencies identified by the Emergency Medicine Milestones Project. The Milestones are intended as a skill development framework, not learning objectives. Both our objectives and the Milestones address dosing of analgesia. In contrast to the Milestones, which focuses heavily on medications and dosing, our objectives provide equal weight to systems-based practice issues and patient communication. The Milestones have a strong focus on procedural sedation (4/11 proficiencies) which we do not address at all. Use of our learning objectives would help learners advance in the pain-related proficiencies described by the Milestones, with the exception of the procedural sedation.

Perhaps the most important difference of our objectives with Milestones and the Model is that our learning objectives specifically mention communicating with patients regarding the risk of addiction. As we confront the opioid epidemic, we must consider the role that prescribed opioids play and discuss this frankly with our patients.

Following creation of the learning objectives, further study should investigate educational outcomes of the resultant teaching module. Do learners gain medical knowledge from the teaching module? Do learners find the knowledge relevant to their clinical practice of EM? Do the teaching modules seem more onerous or enjoyable when compared to traditional education from a live instructor? More importantly, we should address clinical outcomes. Do learners who take these modules administer fewer opioids? Do their patients that report successful treatment of their pain?

Emergency medicine specialists engage with other medical specialties on a daily basis. When collaboration is effective, education between specialists occurs 
in a bidirectional fashion; we teach our consultants and they teach us. The Delphi method allows us to incorporate ideas from other specialist to the benefit of our trainees and, ultimately, our patients.

We thank Michael Clark, MD, MPH, Brenna Farmer, MD, Julianna Jung, MD, Lewis Nelson, MD, and Glenn Treisman, $\mathrm{MD}, \mathrm{PhD}$, for devoting time and expertise to this project.

\section{REFERENCES}

1. Institute of Medicine (US) Committee on Advancing Pain Research, Care, and Education. Relieving Pain in America: A Blueprint for Transforming Prevention, Care, Education, and Research. Washington, DC: National Academies Press, 2011.

2. Centers for Disease Control. National Center for Health Statistics: Emergency Department Visits. Available at: http://www.cdc.gov/nchs/fastats/emergency-department. htm. Accessed Sep 27, 2017.

3. Downey LV, Zun LS. Pain management in the emergency department and its relationship to patient satisfaction. J Emerg Trauma Shock 2010;3:326-30.

4. Gaskin D, Richard P. The economic costs of pain in the United States. J Pain 2012;13:715-24.

5. Han B, Compton WM, Blanco C, Crane E, Lee J, Jones CM. Prescription opioid use, misuse, and use disorders in U.S. adults: 2015 National Survey on Drug Use and Health. Ann Intern Med 2017;167:293-301.

6. Lebovits A, Florence I, Bathina R, Hunko V, Fox MT, Bramble CY. Pain knowledge and attitudes of healthcare providers: practice characteristic differences. Clin J Pain 1997; 13:237-243.

7. Rupp T, Delaney K. Inadequate analgesia in emergency medicine. Ann Emerg Med 2004:43:494-503.

8. Counselman FL, Babu K, Edens MA, et al. The 2016 model of the clinical practice of emergency medicine. J Emerg Med 2017;52:846-9.

9. The Accreditation Council of Graduate Medical Education and The American Board of Emergency Medicine. Emergency Medicine Milestones Project. Available at: https:// www.abem.org/public/docs/default-source/migrated-doc uments-and-files/em-milestones.pdf?sfvrsn=6. Accessed Dec 23, 2017.

10. Dehon E, Jones J, Puskarich M, Sandifer JP, Sikes K. The use of emergency medicine milestones as items on end-ofshift evaluations results in overestimates of residents' proficiency level. J Grad Med Educ 2015;7:192-6.

11. Goldflam K, Bod J, Della-Giustina D, Tsyrulnik A. Emergency medicine residents consistently rate themselves higher than attending assessments on ACGME milestones. West J Emerg Med 2015;16:931-5.

12. National Institutes of Health. NIH Pain Consortium. Available at: http://painconsortium.nih.gov. Accessed on Sep 27, 2017.

13. Polomano R, Dunwoody C, Krenzischek D, et al. Perspective on pain management in the 21 st century. Pain Manag Nurs 2008;9:3-10.

14. Foster MJ, Shurtz S, Pepper C. Evaluation of best practices in the design of online evidence-based practice instructional modules. J Med Libr Assoc 2014;102:3140.

15. Kemper KJ, Gardiner P, Gobble J, Mitra A, Woods C. Randomized controlled trial comparing four strategies for delivering ecurriculum to health care professionals. BMC Med Educ 2006;6:2.

16. Brown B. Delphi Process: A Methodology Used for the Elicitation of Opinions of Experts: An earlier paper published by RAND. 1968. Document No: P-3925

17. Loo R. The Delphi method: a powerful tool for strategic management. Policing: An International Journal of Police Strategies \& Management 2002;25:762-9.

18. Boulkedid R, Abdoul H, Loustau M, et al. Using and reporting the Delphi method for selecting healthcare quality indicators: a systematic review. PLoS one 2011;6: e20476.

19. Cuhls K. Delphi Method. In: Cuhls K, editor. Delphi Surveys. Teaching material for UNIDO foresight seminars. Vienna, Austria: UNIDO, 2005.

20. Atkins RB, Tolson H, Cole BR. Stability of response characteristics of a Delphi panel: application of bootstrap data expansion. BMC Med Res Methodol 2005;1:37. 